\title{
Significance of Development and Reversion of Collaterals on MRI in Early Neurologic Improvement and Long-Term Functional Outcome after Intravenous Thrombolysis for Ischemic Stroke
}

M. Ichijo, E. Iwasawa, Y. Numasawa, K. Miki, S. Ishibashi, M. Tomita, H. Tomimitsu, T. Kamata, H. Fujigasaki, S. Shintani, and H. Mizusawa

\begin{abstract}
BACKGROUND AND PURPOSE: Predicting response to rtPA is essential in the era of endovascular therapy for stroke. The purpose of this study was to elucidate prognostic factors of early neurologic improvement and long-term outcome with respect to the development and reversion of leptomeningeal collaterals in recanalization therapy after acute ischemic stroke.
\end{abstract}

MATERIALS AND METHODS: We analyzed consecutive patients with proximal MCA occlusion treated with rtPA from 2007 to 2012 at 2 hospital stroke centers. All patients routinely underwent brain MR imaging before rtPA. To assess the reversion of collateral signs, we included patients who underwent follow-up MR imaging. We assessed the development and reversion of collaterals by using a combination of 2 MR imaging collateral markers, the hyperintense vessel sign and the posterior cerebral artery laterality sign. Early neurologic improvement was defined as a decrease in the NIHSS score of $\geq 10$ or a score of $\leq 2$ at 24 hours of treatment.

RESULTS: Early neurologic improvement was observed in 22 of 48 eligible patients. The development of collaterals at arrival (15/22 versus $9 / 26, P=.042$ ) was significantly associated with early neurologic improvement. Multivariate analysis adjusting for other variables showed that the development of collaterals at arrival (OR, 4.82; 95\% Cl, 1.34-19.98; $P=.015)$ was independently associated with early neurologic improvement. Reversion of collaterals was significantly associated with successful recanalization $(P<.001)$, and multivariate analysis showed that the reversion of collaterals was an independent prognostic factor of long-term functional outcome (OR, 5.07; $95 \% \mathrm{Cl}$, $1.38-22.09 ; P=.013)$.

CONCLUSIONS: Our results indicate that the development of leptomeningeal collaterals plays a crucial role in achieving early neurologic improvement, and reversion of collaterals predicts a favorable outcome via arterial recanalization after rtPA treatment for acute stroke.

ABBREVIATIONS: $\mathrm{ENI}=$ early neurologic improvement; $\mathrm{HV}=$ hyperintense vessel; IQR = interquartile range; $\mathrm{MCA}=$ middle cerebral artery; $\mathrm{PCA}=$ posterior cerebral artery; TIMI $=$ Thrombolysis in Myocardial Infarction

$\mathbf{P}$ roximal intracranial arterial occlusion is associated with poor functional outcome, and salvaging brain tissue at risk of infarction is of great interest. Intravenous administration of recombinant tissue plasminogen activator to recanalize the occluded artery has significantly reduced mortality and improved longterm outcomes after ischemic stroke, as shown in large clinical trials. ${ }^{1,2}$ Approximately $25 \%$ of patients who received IV rtPA

Received October 8, 2014; accepted after revision March 3, 2015.

From the Department of Neurology and Neurological Science (M.I., E.I., Y.N., S.I., and H.M.), Department of Endovascular Surgery (K.M.), and Clinical Research Center (M.T.), Tokyo Medical and Dental University, Tokyo, Japan; Department of Neurology, JA Toride Medical Center (H.T., S.S.), Ibaraki, Japan; Department of Neurology, Musashino Red Cross Hospital (M.I., T.K.), Tokyo, Japan; and Department of Internal Medicine (H.F.), Metropolitan Bokutoh Hospital, Tokyo, Japan.

Please address correspondence to S. Ishibashi, MD, PhD, Department of Neurology and Neurological Science, Tokyo Medical and Dental University, 1-5-45, Yushima, Bunkyo-ku, Tokyo 113-0034; e-mail: t-ishibashi.nuro@tmd.ac.jp

三 Indicates article with supplemental on-line appendix and table.

http://dx.doi.org/10.3174/ajnr.A4384 experienced neurologic improvement within 24 hours after administration (early neurologic improvement $[\mathrm{ENI}]$ ), ${ }^{3-7}$ and the long-term functional outcome at 3 months was better than that of patients who did not experience ENI. ${ }^{6,8}$ Identifying factors predicting neurologic improvement shortly after reperfusion therapy with rtPA in clinical practice may help select patients who are likely to respond to IV rtPA and improve the selection of patients for more aggressive therapies, including endovascular intervention. ENI has been associated with lower baseline NIHSS scores ${ }^{7}$ and younger age, ${ }^{4,6,7}$ but not with time from onset to treatment. ${ }^{4,7,8}$ Although the theory of thrombolysis predicts that early recanalization should contribute to ENI, a proportion of patients with early recanalization did not experience ENI in some studies, ${ }^{9,10}$ suggesting that other unspecified factors also play a role.

At the acute phase of proximal MCA occlusion, the primary collateral circulation is occasionally established via leptomeningeal anastomoses from the anterior cerebral artery and posterior cerebral artery (PCA). Hyperintense vessels (HVs) on FLAIR MR 


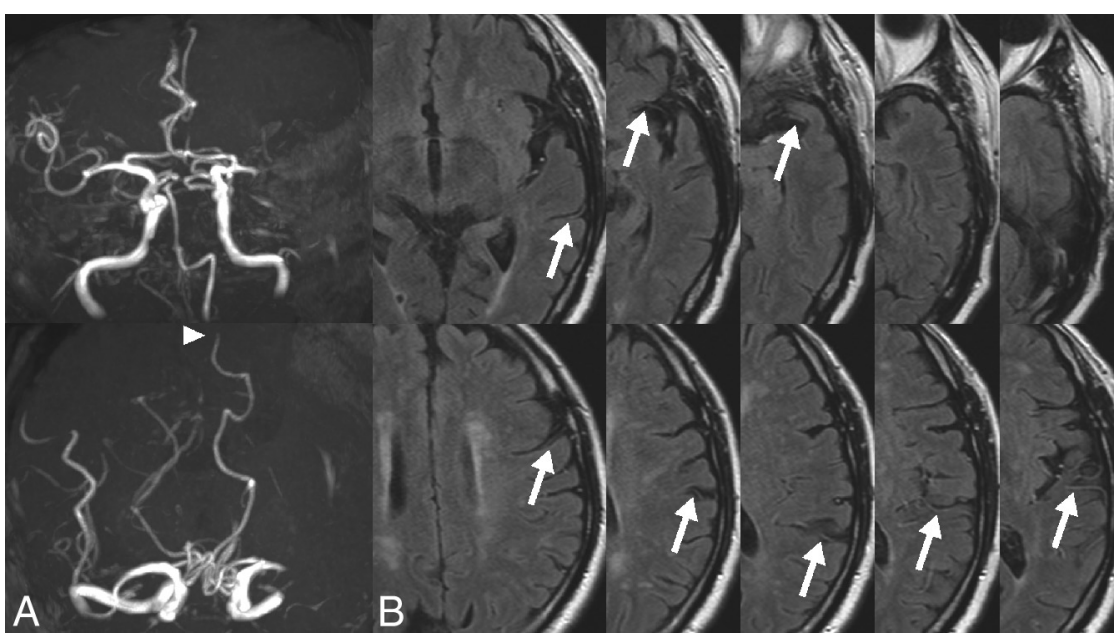

FIG 1. PCA laterality sign and hyperintense vessels on MR images. $A$, MRA shows an occlusion of the M1 portion of the left MCA and signal extent of the ipsilateral PCA (arrowhead). B, FLAIR MR imaging shows hyperintense vessels (arrows) in 8 of 10 axial sections.

imaging $^{11-14}$ and prominent PCA laterality on 3D-TOF MRA ${ }^{15,16}$ were reported as MR imaging radiologic markers of collateral flow. The presence of good collateral circulation before recanalization therapy is correlated with smaller infarct volume and better long-term neurologic outcome in patients with acute ischemic stroke caused by proximal MCA occlusion ${ }^{17}$ as well as with a prolonged therapeutic time window. ${ }^{18}$ Recently, the disappearance of collateral perfusion signs (collateral signs) on MR imaging was reported in patients with internal carotid artery occlusion in whom a high TICI flow grade was restored after immediate endovascular therapy. ${ }^{19}$ This finding indicates that the reversion of collateral signs may be a marker of hemodynamic improvement after recanalization. ${ }^{19}$ In this study, we focused on the development and reversion of leptomeningeal collateral circulation in acute proximal MCA occlusion and investigated their relationship with ENI and long-term outcome in the setting of thrombolytic therapy.

\section{MATERIALS AND METHODS}

For detailed methods, see the On-line Appendix. This retrospective case control study was performed on all patients admitted to 2 hospital stroke centers from April 2007 to November 2012. We selected patients with acute proximal MCA occlusion (M1 or M2 segment) who were treated with IV rtPA according to Japanese guidelines. ${ }^{20}$ All patients routinely underwent brain MR imaging (Signa HDxt 1.5T Optima Edition; GE Healthcare, Milwaukee, Wisconsin; Magnetom Avanto; Siemens, Erlangen, Germany; or Symphony; Siemens) before rtPA and follow-up brain CT after stroke (median, 9 days; interquartile range [IQR], 6-12 days). To assess the reversion of collateral signs, we included patients who underwent follow-up MR imaging. In accordance with the standard protocol of our institutions for patients with ischemic stroke, demographic data and information on cardiovascular risk factors and medical history, the results of diagnostic tests, NIHSS scores, and modified Rankin Scale scores at 3 months after stroke were collected. We assessed ENI, defined as a decrease in NIHSS score of $\geq 10$ or an NIHSS score $\leq 2$ at 24 hours after rtPA treatment. ${ }^{4-8}$

Readers (E.I. and M.I.) blinded to all clinical information as- sessed the presence of PCA laterality and HVs on FLAIR. PCA laterality was considered present if $\geq 1$ segmental extent was observable on axial stereoscopic images (Fig 1A), as described in previous studies. ${ }^{15,16}$ If signal from both PCAs ended in the same segment, laterality was defined as negative. HVs were defined as linear or serpentine hyperintense signals relative to gray matter distal to the Sylvian fissure. ${ }^{12}$ To quantify the degree of HV, 10 FLAIR MR imaging sections were analyzed, from 2 sections below to 7 sections above the first M1 segment in which the MCA appeared (Fig $1 B) .{ }^{14}$ The resulting $\mathrm{HV}$ score ranged from 0 to 10. A large reduction in the HV score was defined as a decrease of $\geq 5$ on follow-up MR imaging. The "development of collaterals” was defined as positive PCA laterality and an HV score of $\geq 5$ on initial MR imaging. The disappearance of PCA laterality or the reduction of the HV score by $\geq 5$ on follow-up MR imaging or both was defined as the "reversion of collaterals." Interrater reliability for the presence of PCA laterality and HV score grading between 2 observers were estimated; in the event of discrepancies between readers, the final result was reached by consensus. Recanalization status after IV rtPA was assessed with a modified grading system based on the Thrombolysis in Myocardial Infarction (TIMI) grade. ${ }^{21}$ Successful recanalization was defined as TIMI 3 on follow-up MRA, and the percentage of successful recanalization was used as the recanalization rate. The Alberta Stroke Program Early CT Score ${ }^{22}$ was used to evaluate the initial DWI hyperintensity and final infarct extent on follow-up CT scans. Initial DWI volume was measured by using $\mathrm{NIH}$ Image (http://rsb.info.nih.gov/nih-image/). Univariable parametric and nonparametric comparisons of clinical characteristics were performed as appropriate. To identify independent predictors of ENI and long-term functional outcome, we performed multivariate logistic regression analyses as shown in the On-line Appendix.

\section{RESULTS}

\section{Baseline Clinical and Radiologic Characteristics in Patients with Collateral Development and Reversion}

From April 2007 to November 2012, 57 patients were examined for inclusion in this study; 5 were excluded for motion artifacts on MR imaging and 4 were excluded because they did not provide informed consent. Among 48 patients who met the inclusion criteria, the median age was 79 years (IQR, $70-83$ years), $46 \%$ were women, and the median initial NIHSS score was 16 (IQR, 11-23). The location of the arterial occlusion was the M1 MCA in 35 patients and the M2 MCA in 13 patients. Development of collaterals (positive PCA laterality and HV score $\geq 5$ at arrival) was observed in $24(50 \%)$ of 48 patients. Twenty-five patients had PCA laterality at arrival. The signal extent of PCA in all these patients was confined to the ipsilateral side of the ischemic hemisphere (Fig 2A). Thirty-nine patients had HV scores of $\geq 5$, and 


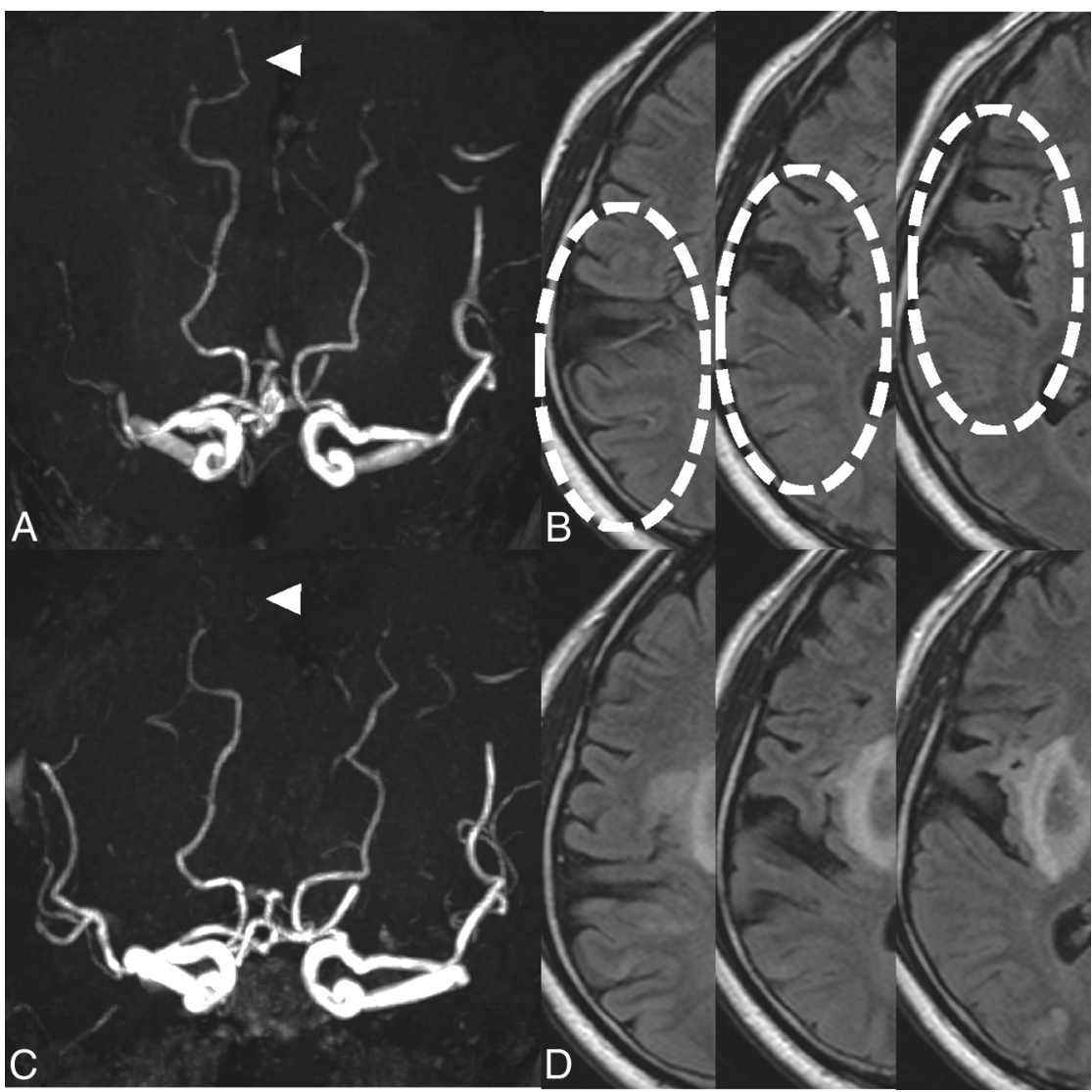

FIG 2. Reversion of collateral signs on MR images. MRA ( $A$ and $C$ ) and FLAIR MR imaging ( $B$ and $D$ ) of a representative patient who experienced early neurologic improvement after IV rtPA. PCA laterality (arrowheads) and hyperintense vessels (dotted circles) were observed before treatment ( $A$ and $B$ ) but disappeared after thrombolysis ( $C$ and $D$ ), indicating the reversion of collaterals.
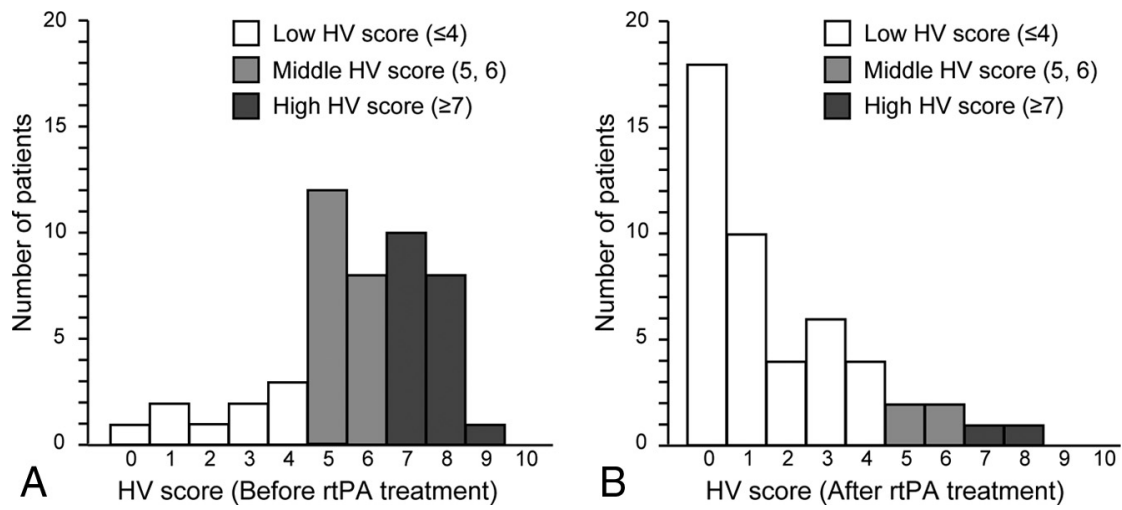

FIG 3. Distribution of the hyperintense vessel (HV) score before and after rtPA treatment. Most patients (39 of $48,81 \%$ ) were initially classified in the middle and high HV score group before treatment $(A)$; however, the number of patients with a middle or high HV score dramatically decreased after thrombolysis $(B)$.

the distribution of HV scores before IV rtPA is shown in Fig $3 A$. The $\kappa$ coefficient for interobserver agreement was 0.917 for PCA laterality and 0.772 for the grading as low, medium, or high $\mathrm{HV}$ score by the 2 observers, which indicated good agreement. Reversion of collaterals (disappearance of PCA laterality and/or a large reduction in the HV score) was observed in 25 (52\%) of 48 patients. The collateral reversion was observed in approximately $50 \%$ of patients in each collateral marker. PCA laterality had disappeared in 13 (52\%) of 25 patients (Fig 2C), and 22 (56\%) of 39 patients showed a large reduction in the HV score $(\geq 5)$ after IV rtPA (Fig $2 B$, $-D)$. The HV score was significantly lower after IV rtPA than before IV rtPA (1 versus 6 ; IQR, $0-3$ versus $5-7 ; P<$ .0001 ; Fig 3B).

The groups with and without reversion of collaterals were equivalent in age, NIHSS score at arrival, cardiovascular risk factors, stroke etiology, and past medication (Table 1). Both groups were equivalent in the duration between the 2 MR imaging scans. The initial NIHSS score, DWI ASPECTS, and DWI volume did not differ significantly between the groups. The NIHSS score obtained 24 hours and 7 days after admission was significantly lower in patients with reversion of collaterals than in those without it (24 hours, 7 versus 11; IQR, 2-12 versus $6-17 ; P=.022 ; 7$ days, 4 versus 8 ; IQR, $1-8$ versus $3-14 ; P=.008$ ). The successful recanalization rate (TIMI 3) assessed by follow-up MR imaging was significantly higher in patients with reversion of collaterals than in those without it (23 of 25 patients versus 8 of 23 patients; $P<.001)$. The follow-up CT ASPECTS was significantly higher in patients with reversion of collaterals than in those without it (8 versus 6; IQR, 6.5-9 versus $4-8 ; P=.017)$, indicating a smaller infarct extent (Table 1). Significantly more patients with reversion of collaterals had a favorable long-term functional outcome (mRS, $0-1$ at 3 months) than those without it (16 of 25 patients versus 8 of 23 patients; $P=$ $.043)$.

\section{Prognostic Factors of ENI and Long- Term Functional Outcome after IV rtPA in Patients with Proximal MCA Occlusion}

ENI was observed in 22 (46\%) of 48 patients. The group with ENI and the group without ENI were equivalent in age, NIHSS score at arrival, cardiovascular risk factors, stroke etiology, and past medication (Table 2). DWI ASPECTS at arrival (8 versus 8; IQR, 6.75-9 versus 6.75-8; $P=.33)$ and the arterial occlusion site $(P=.33)$ did not differ between groups. Consistent with previous research, ${ }^{3-5,7}$ the rate of good long-term functional outcome (modified Rankin Scale score of $0-1$ at 3 months) was significantly higher in the ENIpositive group than the ENI-negative group (16 of 22 patients versus 8 of 26 patients; $P=.008$ ). The development of collateral signs was detected in a significantly greater number of patients in 
Table 1: Characteristics of patients with and without reversion of collaterals ${ }^{a}$

\begin{tabular}{|c|c|c|c|}
\hline & \multicolumn{2}{|c|}{ Reversion of Collaterals } & \multirow[b]{2}{*}{$P$ Value } \\
\hline & Yes $(n=25)$ & No $(n=23)$ & \\
\hline Age (median) (IQR) & $78(71-81)$ & $79(68-86)$ & .52 \\
\hline Male sex (No.) (\%) & $13(52)$ & $13(57)$ & .78 \\
\hline mRS 0-1 before stroke (No.) (\%) & $25(100)$ & $22(96)$ & .48 \\
\hline \multicolumn{4}{|l|}{ Cardiovascular risk factors (No.) (\%) } \\
\hline Hypertension & $15(60)$ & $13(57)$ & 1 \\
\hline Diabetes mellitus & $7(28)$ & $3(13)$ & .29 \\
\hline Hyperlipidemia & $4(16)$ & $7(30)$ & .31 \\
\hline Atrial fibrillation & $21(84)$ & $13(57)$ & .057 \\
\hline Congestive heart failure & $5(20)$ & $4(17)$ & 1 \\
\hline Previous stroke & $6(24)$ & $6(26)$ & 1 \\
\hline Smoking & $9(36)$ & $9(41)$ & .77 \\
\hline \multicolumn{4}{|l|}{ Past medication at stroke onset (No.) (\%) } \\
\hline Antiplatelet therapy & $7(28)$ & $8(35)$ & .76 \\
\hline Anticoagulant therapy & $5(20)$ & $4(17)$ & 1 \\
\hline Antihypertensive therapy & $13(52)$ & $10(44)$ & .58 \\
\hline Statin therapy & $2(8)$ & $4(17)$ & .41 \\
\hline \multicolumn{4}{|l|}{ Stroke etiology (No.) (\%) } \\
\hline Cardioembolism & $19(76)$ & $11(48)$ & .07 \\
\hline Atherosclerosis & $4(16)$ & $8(35)$ & .19 \\
\hline Other or undetermined & $2(8)$ & $4(17)$ & .41 \\
\hline \multicolumn{4}{|l|}{ Severity of stroke at arrival (median) (IQR) } \\
\hline Initial GCS & 13 (11-14) & $12(10-14)$ & .62 \\
\hline Initial DWI volume (mL) & $21.6(13.8-42.5)$ & $22.9(10.6-41.1)$ & .63 \\
\hline DWI ASPECTS at arrival & $8(7-9)$ & $8(6-8)$ & .72 \\
\hline Initial NIHSS score & $17(14-24)$ & $16(11-21)$ & .35 \\
\hline Duration between 2 MRI scans (days) & $7(5-9)$ & $6(3-8)$ & .2 \\
\hline \multicolumn{4}{|l|}{$\begin{array}{l}\text { Neurologic and radiologic outcome } \\
\text { after rtPA (median) (IQR) }\end{array}$} \\
\hline 24-hr NIHSS & $7(2-12)$ & $11(6-17)$ & $.022^{\mathrm{b}}$ \\
\hline 7-day NIHSS & $4(1-8)$ & $8(3-14)$ & $.008^{\mathrm{c}}$ \\
\hline Hemorrhagic transformation & $6(24)$ & $10(43)$ & .22 \\
\hline Successful recanalization & $23(92)$ & $8(35)$ & $<.001^{\mathrm{d}}$ \\
\hline Follow-up CT ASPECTS & $8(6.5-9)$ & $6(4-8)$ & $.017^{\mathrm{b}}$ \\
\hline $\mathrm{M} 1$ to $\mathrm{M} 6$ area in ASPECTS & $5(4-6)$ & $3(2-6)$ & $.021^{\mathrm{b}}$ \\
\hline $\mathrm{C}, \mathrm{I}, \mathrm{L}, \mathrm{IC}$ area in ASPECTS & $3(2.5-4)$ & $3(2-3)$ & .12 \\
\hline mRS 0-1 at $3 \mathrm{mo}(\mathrm{No}).(\%)$ & $16(64)$ & $8(35)$ & $.043^{b}$ \\
\hline
\end{tabular}

Note:-GCS indicates Glasgow Coma Scale; C, caudate nucleus; I, insular cortex; L, lenticular nucleus; IL, internal capsule.

${ }^{a}$ For continuous variables, the median and $P$ values (Mann-Whitney $U$ test) are shown. The resulting proportions and the $P$ values (Fisher exact test with Yates correction, when appropriate) are shown

${ }^{\mathrm{b}} P<.05$.

${ }^{c} P<.01$.

${ }^{\mathrm{d}} P<.001$ was considered significant

the group with ENI than in the group without ENI (15 of 22 patients versus 9 of 26 patients, $P=.042$ ).

Univariate analysis revealed that the history of atrial fibrillation and the development of collaterals at arrival were significantly associated with ENI (Table 3). Multivariate logistic regression analysis was performed to further evaluate the independent predictors of ENI after adjusting for age, history of atrial fibrillation, NIHSS score at arrival, and time to rtPA administration as covariates. The development of collaterals at arrival (OR, 4.82; 95\% CI, 1.34-19.98; $P=.015)$ and history of atrial fibrillation (OR, 5.32; 95\% CI, 1.16-32.1; $P=.031$ ) were independently associated with ENI after adjustment for other variables (Table 3). Univariate and multivariate analyses were performed to evaluate the prognostic factors for long-term functional outcome. In a univariate analysis, initial DWI volume and reversion of collaterals were predictive of long-term functional outcome. Multivariate logistic regression analysis was performed to further evaluate independent predictors of clinical outcome. Reversion of collaterals
(OR, 5.07; 95\% CI, 1.38-22.09; $P=$ .013 ) was independently associated with favorable outcome, after adjustment for other variables (On-line Table).

\section{DISCUSSION}

We showed that the development of leptomeningeal collaterals, assessed by PCA laterality on MRA and HV score on FLAIR, was significantly associated with ENI in patients with proximal MCA occlusion treated with IV rtPA. Because the principal purpose of thrombolytic therapy is to open obstructed vessels and to reperfuse the ischemic penumbra, ${ }^{23}$ early recanalization after IV rtPA should contribute to ENI. However, 37\%-46\% of patients with recanalization performed within 2 hours after IV rtPA, as assessed by transcranial Doppler, ${ }^{9}$ or after endovascular treatment ${ }^{10}$ showed no immediate clinical improvement or may have even worsened. In our study, the positive association observed between the development of collateral signs on MR imaging and ENI indicates that leptomeningeal collateral development may be an important factor for achieving ENI after recanalization therapy.

Several factors may explain why welldeveloped collaterals lead to ENI. First, the leptomeningeal collateral circulation plays an important role in preserving cerebral blood flow in the territory of the occluded artery. ${ }^{24,25}$ Well-developed leptomeningeal collateral circulation is associated with maintaining the perfusion of penumbral regions, as evaluated by using CT perfusion or MR imaging perfusion methods, ${ }^{26,27}$ resulting in the protection of distal brain tissues. Consistently, our recent study showed that patients with PCA laterality had a significantly smaller infarct volume, particularly in the cortical region, on follow-up CT than those without this sign who were treated with rtPA after acute MCA occlusion. ${ }^{16}$ Second, retrograde collateral filling may allow the access of thrombolytic agents to distal clots, ${ }^{28}$ which may minimize the ischemic damage to the structure of the distal vessels themselves. Because cerebral arteries and capillaries in the brain are impaired early and in a progressive fashion after focal cerebral ischemia, ${ }^{29,30}$ the degree of ischemic vascular injury can be minimized by collateral blood supply to vessels and brain tissue located within the territory of the occluded artery. These possible effects of collateral circulation may protect brain tissue and/or vessel structures from ischemic damage until MCA recanalization.

Another important finding of our study was that the collateral signs observed on MR imaging were diminished in almost half of 
Table 2: Comparison of the presence and absence of early neurologic improvement after IV rtPA in patients with proximal middle cerebral artery occlusion ${ }^{a}$

\begin{tabular}{|c|c|c|c|}
\hline & \multicolumn{2}{|c|}{ Early Neurologic Improvement } & \multirow[b]{2}{*}{$P$ Value } \\
\hline & Yes $(n=22)$ & No $(n=26)$ & \\
\hline Age (yr) ( median) (IQR) & $78.5(74-81)$ & $78.5(68-84)$ & .92 \\
\hline Male sex (No.) (\%) & $10(45)$ & $16(62)$ & .38 \\
\hline mRS 0-1 before stroke (No.) (\%) & $22(100)$ & $25(96)$ & 1 \\
\hline NIHSS score at arrival (median) (mean) & $17.9 \pm 8.1$ & $16.0 \pm 5.7$ & .29 \\
\hline Systolic blood pressure (mean) & $161.3 \pm 29.6$ & $155.0 \pm 30.8$ & .91 \\
\hline Diastolic blood pressure (mean) & $88.5 \pm 26.1$ & $78.3 \pm 21.9$ & .19 \\
\hline Temperature $\left({ }^{\circ} \mathrm{C}\right)($ mean) & $36.3 \pm 0.4$ & $36.2 \pm 0.7$ & .75 \\
\hline \multicolumn{4}{|l|}{ Cardiovascular risk factors (No.) (\%) } \\
\hline Hypertension & $14(64)$ & $14(54)$ & .57 \\
\hline Diabetes mellitus & $7(27)$ & $4(15)$ & .48 \\
\hline Hyperlipidemia & $5(23)$ & $6(23)$ & 1 \\
\hline Atrial fibrillation & $19(86)$ & $15(58)$ & .054 \\
\hline Congestive heart failure & $4(18)$ & $5(19)$ & 1 \\
\hline Previous stroke & $5(23)$ & $7(27)$ & 1 \\
\hline Smoking & $6(27)$ & $12(48)$ & .23 \\
\hline \multicolumn{4}{|l|}{ Past medication at stroke onset (No.) (\%) } \\
\hline Antiplatelet therapy & $8(36)$ & $7(27)$ & .54 \\
\hline Anticoagulant therapy & $4(18)$ & $5(19)$ & 1 \\
\hline Antihypertensive therapy & $14(64)$ & $9(35)$ & .08 \\
\hline Statin therapy & $2(9)$ & $4(15)$ & .67 \\
\hline \multicolumn{4}{|l|}{ Stroke etiology (No.) (\%) } \\
\hline Cardioembolism & $17(77)$ & $13(50)$ & .074 \\
\hline Atherosclerosis & $4(18)$ & $8(31)$ & .5 \\
\hline Other or undetermined & $1(5)$ & $5(19)$ & .2 \\
\hline \multicolumn{4}{|l|}{ Imaging analysis } \\
\hline Initial DWI volume (mL) (median) (IQR) & $19.8(11.5-42.3)$ & $22.7(13.9-41.2)$ & .26 \\
\hline DWI ASPECTS at arrival (median) (IQR) & $8(6.75-9)$ & $8(6.75-8)$ & .33 \\
\hline MCA Ml occlusion (No.) (\%) & $18(82)$ & $17(65)$ & .33 \\
\hline Development of collaterals at arrival (No.) (\%) & $15(68)$ & $9(35)$ & $.042^{b}$ \\
\hline \multicolumn{4}{|l|}{ Stroke outcome } \\
\hline Follow-up CT ASPECTS (median) (IQR) & $8(6-9.25)$ & $6(4.75-8)$ & $.004^{d}$ \\
\hline mRS 0-1 at $3 \mathrm{mo}$ (No.) (\%) & $16(73)$ & $8(31)$ & $.008^{\mathrm{d}}$ \\
\hline
\end{tabular}

${ }^{a}$ For continuous variables, the median and $P$ values (Mann-Whitney $U$ test) are shown. The resulting proportions and the $P$ values (Fisher exact test with Yates correction, when appropriate) are shown.

$\mathrm{b} P<.05$.

c The development of collaterals was defined as positive in the presence of PCA laterality and an HV score of $\geq 5$ on initial MRI.

${ }^{d} P<.01$ was considered significant.

Table 3: Univariate analyses and multivariate logistic regression analysis for the association of early neurologic improvement after IV rtPA in patients with proximal middle cerebral artery occlusion

\begin{tabular}{|c|c|c|c|c|}
\hline & \multirow{2}{*}{$\begin{array}{r}\text { Crude OR } \\
(95 \% \mathrm{CI})\end{array}$} & \multirow[b]{2}{*}{$P$ Value } & \multirow{2}{*}{$\frac{\text { Adjusted OR }}{(95 \% \mathrm{Cl})}$} & \multirow[b]{2}{*}{$P$ Value } \\
\hline & & & & \\
\hline Age $(y r)$ & $0.99(0.95-1.06)$ & .91 & $1.00(0.94-1.06)$ & .95 \\
\hline Male sex & $0.52(0.16-1.63)$ & .26 & & \\
\hline History of atrial fibrillation & $4.64(1.20-23.36)$ & $.025^{\mathrm{a}}$ & $5.32(1.16-32.1)$ & $.031^{\mathrm{a}}$ \\
\hline NIHSS score at arrival & $1.04(0.88-1.04)$ & .34 & $1.02(0.89-1.09)$ & .72 \\
\hline DWI ASPECTS at arrival & $1.16(0.80-1.73)$ & .44 & & \\
\hline Time to rtPA administration & $0.99(0.97-1.02)$ & .49 & $1.00(0.97-1.03)$ & .84 \\
\hline Development of collaterals at arrival ${ }^{\mathrm{b}}$ & $4.0(1.25-14.27)$ & $.019^{\mathrm{a}}$ & $4.82(1.34-19.98)$ & $.015^{\mathrm{a}}$ \\
\hline
\end{tabular}

a $P<.05$ was considered significant.

${ }^{\mathrm{b}}$ The development of collaterals was defined as positive in the presence of PCA laterality positivity and an HV score of $\geq 5$ on initial MRI.

c Adjusted for age, history of atrial fibrillation, NIHSS score at arrival, and time to rtPA administration. for up to 2 weeks. ${ }^{31}$ However, the mechanism underlying these changes in collateral signs has not been well-documented. In the course of ischemic stroke, collateral blood flow should play an important role in providing blood to cerebral tissues at risk of infarction in the territory of the occluded artery. ${ }^{24-27}$ Therefore, a failure or reduction of collateral circulation may cause infarct growth in the occluded territory. In a recent study that evaluated the temporal profile of collateral flow by using modified perfusion MR imaging in patients with acute ischemic stroke, compared with initial MR imaging, the leptomeningeal collateral flow reduced 3-5 days after stroke onset in approximately onethird of patients without spontaneous recanalization. $^{32}$ In the no-recanalization group, the decrease in collateral flow was associated with infarct growth within 5 days. ${ }^{32}$ Therefore, maintaining collateral flow may help prevent infarct growth during the acute phase of ischemic stroke if recanalization is not achieved.

We observed reversion of collaterals after thrombolysis in approximately $50 \%$ of patients with initially well-developed collaterals and significant association with favorable long-term functional outcome. Because early collateral development via leptomeningeal anastomoses after MCA occlusion is induced by a pressure gradient between the anterior cerebral artery or PCA territory and a territory distal to the MCA occlusion site, ${ }^{24,25}$ leptomeningeal collaterals may decrease or disappear after the occluded MCA reopens at an optimal timing because of normalization of the pressure gradient. Several studies in patients with acute internal carotid or MCA occlusion reported that HV collateral signs on initial FLAIR MR imaging disappeared within several days after early spontaneous recanalization ${ }^{31}$ or successful vascularization via endovascular therapy. ${ }^{19}$ In this study, reversion of collaterals was significantly associated with a high rate

patients with well-developed collateral signs before rtPA administration; we found a significant association between the reversion of collateral signs and long-term functional outcome. A previous study reported that $\mathrm{HV}$ s were observed on FLAIR in all patients with MCA occlusion within 24 hours of stroke onset and the percentage of $\mathrm{HV}$-positive patients subsequently decreased in a timedependent manner, though HVs were still observed in some cases of successful recanalization on follow-up MR imaging. Thus, according to these findings, collateral reversion may indicate improvement in cerebral hemodynamic status. Indeed, the extent of infarction in the cortical regions (M1-M6 in ASPECTS; total score, 6) was significantly smaller in patients with collateral reversion than in those without it (median ASPECTS, 5 versus 3; $P=$ .021 ), but no significant differences were observed in other 
ASPECTS regions, such as the insular cortex, basal ganglia, and internal capsule (median ASPECTS, 3 versus 3, $P=.12$ ) (Table 1). Because leptomeningeal collaterals, which are upstream of the penetrating cortical vessels, play a major role in compensating for low blood flow in the cerebral cortex after ischemic stroke, collateral reversion may exhibit the recovery of effective "cortical" blood flow after the optimal timing of recanalization, contributing to prevention of infarct extent.

This study has several limitations. First, it was retrospective and limited by a small sample size and a nonrandomized population; and the timing for initial and follow-up MR imaging was heterogeneous. The timing of the development and reversion of collateral circulation is not well-elucidated. The timing and duration of MR imaging may influence the existence of collateral signs, though there was no significant association between the timing of the MR imaging scan and the collateral development or reversion in our study. Second, MR imaging acquisitions before and after rtPA therapy during acute ischemic stroke may be challenging in a clinical setting. The greatest benefit of rtPA therapy comes from earlier treatment, and MR imaging screening before rtPA may cause a time delay. Generalizing our results to all hospitals may be a problem because the delay in MR imaging screening might be greater in centers less specialized for emergency stroke MR imaging.

\section{CONCLUSIONS}

Recently, the Multicenter Randomized Clinical Trial of Endovascular Treatment for Acute Ischemic Stroke in the Netherlands (MR CLEAN) study reported that endovascular therapy within 6 hours of stroke onset in addition to rtPA improved functional independence at 3 months. ${ }^{33}$ Leptomeningeal collaterals contribute to ENI after acute ischemic stroke, and the development of collateral signs on MR imaging can help identify patients more likely to show ENI in the setting of thrombolysis and help us select patients for additional endovascular therapy. Considering the results, reversion of collaterals is associated with more favorable outcome in patients with acute proximal MCA occlusion after administration of rtPA, which may be due to improved hemodynamic status. Reversion of collaterals may help us to examine the risk of recurrent stroke or infarct extent, to achieve an appropriate prevention after proximal cerebral artery occlusion.

\section{ACKNOWLEDGMENTS}

The authors would like to thank Enago (www.enago.jp) for the English language review.

Disclosures: Tomoyuki Kamata_UNRELATED: Payment for Lectures (including service on Speakers Bureaus): Otsuka Pharmaceutical, Eisai, AstraZeneca, Ono Pharmaceutical, Novartis Pharma, Bayer, Shionogi.

\section{REFERENCES}

1. National Institute of Neurological Disorders and Stroke rt-PA Stroke Study Group. Tissue plasminogen activator for acute ischemic stroke. N Engl J Med 1995;333:1581-87

2. Hacke W, Kaste M, Fieschi C, et al; Second European-Australasian Acute Stroke Study Investigators. Randomised double-blind placebo-controlled trial of thrombolytic therapy with intravenous alteplase in acute ischaemic stroke (ECASS II). Lancet 1998;352:1245-51 3. Felberg RA, Okon NJ, El-Mitwalli A, et al. Early dramatic recovery during intravenous tissue plasminogen activator infusion: clinical pattern and outcome in acute middle cerebral artery stroke. Stroke 2002;33:1301-07

4. Brown DL, Johnston KC, Wagner DP, et al. Predicting major neurological improvement with intravenous recombinant tissue plasminogen activator treatment of stroke. Stroke 2004;35:147-50

5. Saposnik G, Di Legge S, Webster F, et al. Predictors of major neurologic improvement after thrombolysis in acute stroke. Neurology 2005;65:1169-74

6. Blinzler C, Breuer L, Huttner HB, et al. Characteristics and outcome of patients with early complete neurological recovery after thrombolysis for acute ischemic stroke. Cerebrovasc Dis 2011;31:185-90

7. Machumpurath B, Davis SM, Yan B. Rapid neurological recovery after intravenous tissue plasminogen activator in stroke: prognostic factors and outcome. Cerebrovasc Dis 2011;31:278-83

8. Muresan IP, Favrole P, Levy P, et al. Very early neurologic improvement after intravenous thrombolysis. Arch Neurol 2010;67:1323-28

9. Alexandrov AV, Hall CE, Labiche LA, et al. Ischemic stunning of the brain: early recanalization without immediate clinical improvement in acute ischemic stroke. Stroke 2004;35:449-52

10. Penumbra Pivotal Stroke Trial Investigators. The Penumbra Pivotal Stroke Trial: safety and effectiveness of a new generation of mechanical devices for clot removal in intracranial large vessel occlusive disease. Stroke 2009;40:2761-68

11. Schellinger PD, Chalela JA, Kang DW, et al. Diagnostic and prognostic value of early MR imaging vessel signs in hyperacute stroke patients imaged $<3$ hours and treated with recombinant tissue plasminogen activator. AJNR Am J Neuroradiol 2005;26:618-24

12. Lee KY, Latour LL, Luby M, et al. Distal hyperintense vessels on FLAIR: an MRI marker for collateral circulation in acute stroke? Neurology 2009;72:1134-39

13. Liu W, Xu G, Yue X, et al. Hyperintense vessels on FLAIR: a useful non-invasive method for assessing intracerebral collaterals. Eur J Radiol 2011;80:786-91

14. Olindo S, Chausson N, Joux J, et al. Fluid-attenuated inversion recovery vascular hyperintensity: an early predictor of clinical outcome in proximal middle cerebral artery occlusion. Arch Neurol 2012;69:1462-68

15. Uemura A, O'uchi T, Kikuchi Y, et al. Prominent laterality of the posterior cerebral artery at three-dimensional time-of-flight MR angiography in M1-segment middle cerebral artery occlusion. AJNR Am J Neuroradiol 2004;25:88-91

16. Ichijo M, Miki K, Ishibashi S, et al. Posterior cerebral artery laterality on magnetic resonance angiography predicts long-term functional outcome in middle cerebral artery occlusion. Stroke 2013; 44:512-15

17. Bang OY, Saver JL, Kim SJ, et al. Collateral flow predicts response to endovascular therapy for acute ischemic stroke. Stroke 2011;42: 693-99

18. Ribo M, Flores A, Rubiera M, et al. Extending the time window for endovascular procedures according to collateral pial circulation. Stroke 2011;42:3465-69

19. Liu W, Yin Q, Yao L, et al. Decreased hyperintense vessels on FLAIR images after endovascular recanalization of symptomatic internal carotid artery occlusion. Eur J Radiol 2012;81:1595-600

20. Shinohara Y, Yamaguchi T. Outline of the Japanese Guidelines for the Management of Stroke 2004 and subsequent revision. Int $J$ Stroke 2008;3:55-62

21. TIMI Study Group. The Thrombolysis in Myocardial Infarction (TIMI) trial—phase I findings. N Engl J Med 1985;312:932-36

22. Barber PA, Demchuk AM, Zhang J, et al; ASPECTS Study Group. Validity and reliability of a quantitative computed tomography score in predicting outcome of hyperacute stroke before thrombolytic therapy. Lancet 2000;355:1670-74

23. De Silva DA, Fink JN, Christensen S, et al; Echoplanar Imaging Thrombolytic Evaluation Trial (EPITHET) Investigators. Assessing reperfusion and recanalization as markers of clinical outcomes af- 
ter intravenous thrombolysis in the Echoplanar Imaging Thrombolytic Evaluation Trial (EPITHET). Stroke 2009;40:2872-74

24. Shuaib A, Butcher K, Mohammad AA, et al. Collateral blood vessels in acute ischaemic stroke: a potential therapeutic target. Lancet Neurol 2011;10:909-21

25. Liebeskind DS. Collateral circulation. Stroke 2003;34:2279-84

26. Miteff F, Levi CR, Bateman GA, et al. The independent predictive utility of computed tomography angiographic collateral status in acute ischaemic stroke. Brain 2009;132:2231-38

27. Bang OY, Saver JL, Buck BH, et al; UCLA Collateral Investigators. Impact of collateral flow on tissue fate in acute ischaemic stroke. J Neurol Neurosurg Psychiatry 2008;79:625-29

28. Caplan LR, Hennerici M. Impaired clearance of emboli (washout) is an important link between hypoperfusion, embolism, and ischemic stroke. Arch Neurol 1998;55:1475-82
29. Cipolla MJ, McCall AL, Lessov N, et al. Reperfusion decreases myogenic reactivity and alters middle cerebral artery function after focal cerebral ischemia in rats. Stroke 1997;28:176-80

30. del Zoppo GJ, Mabuchi T. Cerebral microvessel responses to focal ischemia. J Cereb Blood Flow Metab 2003;23:879-94

31. Maeda M, Koshimoto Y, Uematsu H, et al. Time course of arterial hyperintensity with fast fluid-attenuated inversion-recovery imaging in acute and subacute middle cerebral arterial infarction. $J$ Magn Reson Imaging 2001;13:987-90

32. Campbell BC, Christensen S, Tress BM, et al; EPITHET Investigators. Failure of collateral blood flow is associated with infarct growth in ischemic stroke. J Cereb Blood Flow Metab 2013;33:1168-72

33. Berkhemer OA, Fransen PS, Beumer D, et al; MR CLEAN Investigators. A randomized trial of intraarterial treatment for acute ischemic stroke. N Engl J Med 2015;372:11-20 